\title{
Monoterpene Glycosides and Triterpene Acids from Eriobotrya deflexa
}

\author{
Tzong-Huei Lee, ${ }^{\dagger}$ Shoei-Sheng Lee, ${ }^{\ddagger}$ Yuh-Chi Kuo, ${ }^{\S}$ and Chang-Hung Chou*,t, \\ Institute of Botany, Academia Sinica, Nankang, Taipe, Taiwan 115, School of Pharmacy, College of Medicine, National \\ Taiwan University, Taipei, Taiwan 100, National Research Institute of Chinese Medicine, Shih-Pai, Taipei, Taiwan 112, and \\ Department of Biological Sciences, National Sun Yat-Sen University, Kaohsiung, Taiwan 804, Republic of China
}

Received J anuary 26, 2001

\begin{abstract}
A phytochemical study on a methanolic extract of leaves of Eriobotrya deflexa led to the isolation and characterization of nine terpenoid compounds. Four of these are new chemical entities, including two monoterpene glycosides, (3S)-O- $\alpha$-L-rhamnopyranosyl-(1 $\rightarrow 3)$-[4-O-(E)-coumaroyl ]- $\alpha$-L-rhamnopyranosyl$(1 \rightarrow 6)$ - $\beta$-D-glucopyranosyl-linalool $(\mathbf{1})$ and (3S)-O- $\alpha$-L-rhamnopyranosyl-(1 $\rightarrow 3$ )-[4-O-(Z)-coumaroyl]- $\alpha$-Lrhamnopyranosyl-( $1 \rightarrow 6)$ - $\beta$-D-glucopyranosyl-linalool (2), and two triterpene acids, 1 $\beta, 2 \alpha, 19 \alpha$-trihydroxy3-oxo-12-ursen-28-oic acid (3) and $2 \alpha, 3 \alpha, 19 \alpha$-trihydroxy-12-ol eanen-28-oic acid (4). Their structures were elucidated on the basis of spectroscopic analysis. The activities of these isolates in an in vitro antiproliferation test were also determined.
\end{abstract}

Eriobotrya deflexa N. (Rosaceae), an erect shrub indigenous to Taiwan, is widely distributed in hardwood forests at about $1500 \mathrm{~m}$ in elevation throughout the island. ${ }^{1}$ Leaves of E. deflexa have been used in traditional Chinese medicine as an expectorant and antitussive. ${ }^{2} \mathrm{~A}$ closely related species, E. japonica, is commonly known as "loquat". ${ }^{3} \mathrm{~A}$ series of sesquiterpene glycosides, ${ }^{4-6}$ triterpene acids, ${ }^{7}$ and triterpene esters 7,8 have been isol ated from $\mathrm{E}$. japonica, and among them, several components were found to show significant bioactivities including antiviral, ${ }^{8}$ antiinflammatory, ${ }^{9}$ and hypoglycemic properties. ${ }^{10,11}$ To determine if E. deflexa can be used as a substitute for E. japonica, its leaf chemical constituents were investigated. Additionally, to determine the immunomodulatory activity of the $E$. deflexa isolates obtained, these substances were evaluated in an in vitro antiproliferation assay.

\section{Results and Discussion}

A methanolic extract of the dried leaves of $\mathrm{E}$. deflexa was fractionated by liquid-liquid partitioning into fractions soluble in n-hexane, chloroform, and n-butanol. The nbutanol- and chloroform-soluble fractions were then subjected to Sephadex LH-20 column chromatography and HPLC to give two monoterpene glycosides ( $\mathbf{1}$ and $\mathbf{2}$ ) and seven triterpene acids (3-9), respectively. Of these, compounds 5-9 were found to be known, based on spectral comparison with reported data. Compounds 5-8 are ursolic acid analogues and were identified as euscaphic acid (5), obtained previously from E. japonica ${ }^{7}$ and Rosa laevigata, ${ }^{12}$ 1 $\beta$-hydroxy-2-oxopomolic acid (6) and 2-oxopomolic acid (7), both isolated from $\mathrm{R}$. woodsi $\mathrm{i}, 13$ and $2 \alpha, 19 \alpha$-dihydroxy-3oxo-12-ursen-28-oic acid (8), a constituent of Geum japonicum. ${ }^{14}$ Compound $\mathbf{9}$ is an ol eanolic acid anal ogue and was identified as arjunic acid, having been isolated from the bark of Terminalia arjuna ${ }^{15}$ and T. macroptera. ${ }^{16}$

The molecular formula for $\mathbf{1}, \mathrm{C}_{37} \mathrm{H}_{54} \mathrm{O}_{16}$, was determined by ${ }^{13} \mathrm{C} N M R$, DEPT, and FABMS data. The ${ }^{1} \mathrm{H}$ NMR spectrum of $\mathbf{1}$ (Table 1) indicated that the molecule

* To whom correspondence should be addressed. Tel: 8862 27899590, ext 451. Fax: 8862 27827954. E-mail: chou@gate.sinica.edu.tw.

† Institute of Botany, Academia Sinica.

₹ School of Pharmacy, National Taiwan University.

$\S$ National Research Institute of Chinese Medicine.

${ }^{\perp}$ Department of Biological Sciences, National Sun Yat-Sen University.

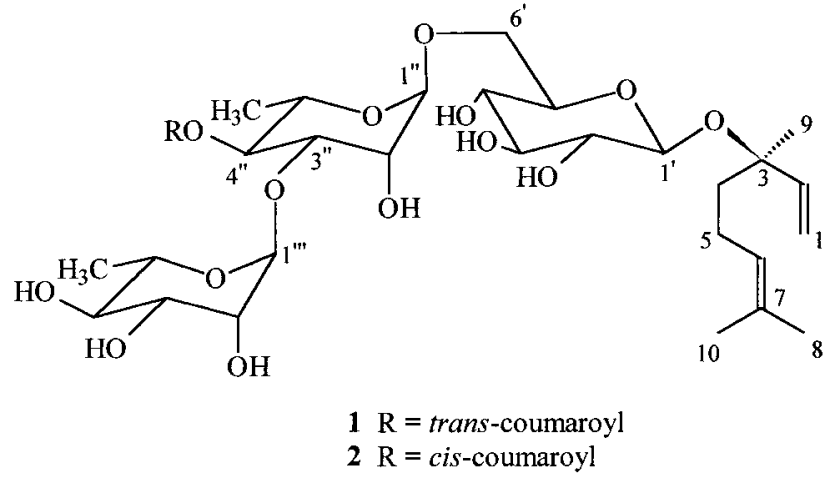

contained three sugar units, as evident from the presence of three anomeric proton signals at $\delta 4.33(\mathrm{~d}, \mathrm{~J}=7.8 \mathrm{~Hz})$, 4.77 (brs), and $4.82(\mathrm{~d}, \mathrm{~J}=1.5 \mathrm{~Hz})$. Of these three sugars, two were assigned as $\alpha$-L-rhamnose units, supported by signals typical for anomeric protons and two secondary methyl signals ( $\delta 1.16$ and $1.24, \mathrm{~d}, J=6.2 \mathrm{~Hz}$ ). Besides the sugar proton regions, the ${ }^{1} \mathrm{H}$ NMR spectrum of $\mathbf{1}$ showed signals for a trans-coumaroyl moiety, ${ }^{8}$ an $A A^{\prime} X X^{\prime}$ system at $\delta 6.80$ (H-6"'"' and $\left.\mathrm{H}-8^{\prime \prime \prime \prime}\right)$ and 7.47 ( $\mathrm{H}-5^{\prime \prime \prime \prime \prime}(\mathrm{OAX}=$ $8.6 \mathrm{~Hz})$, and an AX system at $\delta 6.33\left(\mathrm{H}-2^{\prime \prime \prime \prime}\right)$ and $7.65(\mathrm{H}-$ $\left.3^{\prime \prime \prime \prime}\right)(\mathrm{H}$ trans $=15.9 \mathrm{~Hz})$. In addition, this spectrum also displayed signals for a linalool moiety, ${ }^{17}$ three methyl singlets at $\delta 1.34,1.56$, and 1.61, two methylenes at $\delta 1.62$ and $2.04(2 \mathrm{H}, \mathrm{m})$, and four olefinic protons at $\delta 5.08(1 \mathrm{H}$, $\mathrm{t}, \mathrm{J}=7.0 \mathrm{~Hz}), 5.21(2 \mathrm{H}, \mathrm{m})$, and $6.06(1 \mathrm{H}, \mathrm{dd}, \mathrm{J}=10.7$, $18.0 \mathrm{~Hz}$ ). The presence of this moiety was supported by the ${ }^{13} \mathrm{C}$ NMR and DEPT data, in which the C-3 signal in linal ool was significantly shifted downfield ( $\delta 81.4$ vs 72.9 ), suggesting that the hydroxy group at C-3 is glycosylated. Acid hydrolysis of $\mathbf{1}$ yielded S-(+)-linal ool, ${ }^{18}$ rhamnose, and glucose, with the sugars being analyzed as their TMS derivatives by GC-MS. ${ }^{19}$ The HMBC spectrum of 1 showed the anomeric proton signal $\mathrm{H}_{-1} \mathrm{I}^{\prime}(\mathrm{glc})(\delta 4.33, \mathrm{~d}, \mathrm{~J}=7.8$ $\mathrm{Hz}$ ) coupled to $\mathrm{C}-3$ of linalool $(\delta 81.4, \mathrm{~s})$, the $\mathrm{H}-1^{\prime \prime}$ (rha- 1 ) ( $\delta$ 4.77) signal coupled to C-6' (glc) $(\delta 68.0, \mathrm{t})$, and the H-1"' (rha-2) ( $\delta$ 4.82) signal coupled to C-3" (rha-1) ( $\delta$ 78.5, d). These correlations helped to determine the main part of the structure of $\mathbf{1}$ as (3S)-O- $\alpha$-L-rhamnopyranosyl-(1 $\rightarrow 3$ )$\alpha$-L-rhamnopyranosyl-( $1 \rightarrow 6)-\beta$-D-glucopyranosyllinalool. The coumaroyl moiety in $\mathbf{1}$ was assigned at C-4" of the central 


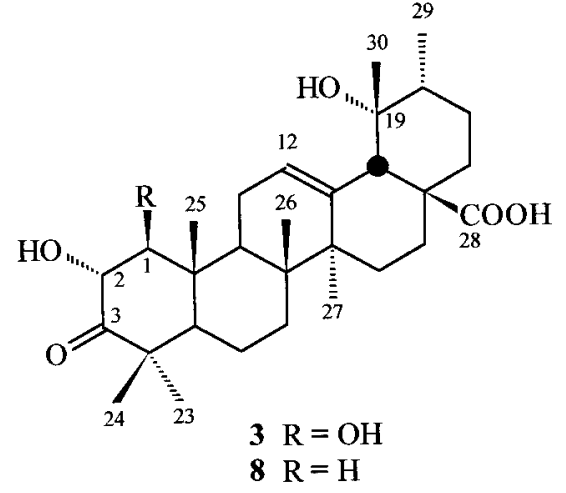<smiles>[R2][R2](=O)O</smiles><smiles>C[C@H]1CCC2(C(=O)O)CC[C@]3(C)C(=CCC4[C@@]5(C)C[C@H](O)[C@@H](O)C(C)(C)C5CC[C@]43C)C2=C1C(C)(O)O</smiles>
5

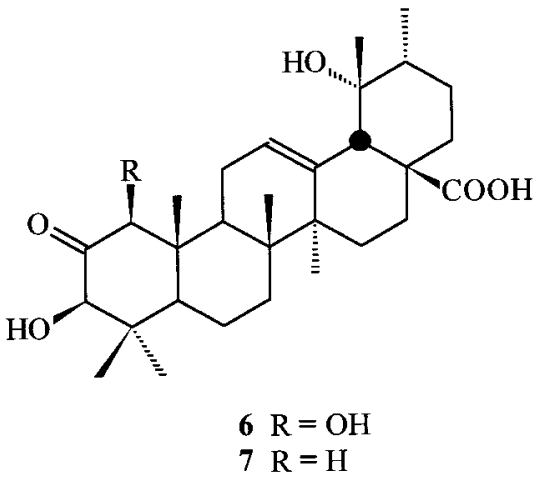

rhamnose residue (rha-1) on the basis of observation of the downfield-shifted signal of the H-4" ( $\delta 5.18$ vs 3.35) with respect to the corresponding signal of the nonsubstituted rhamnose unit (rha-2). This proposal was also confirmed by the HMBC spectrum, which revealed a three-bond coupling between $\mathrm{H}-4^{\prime \prime}$ and $\mathrm{C}-\mathrm{I}^{\prime \prime \prime \prime}$ ( $\delta$ 168.4) of the coumaroyl unit. In addition, this coumaroyl moiety was in the trans form, deduced from the coupling constant between $\mathrm{H}-2^{\prime \prime \prime \prime}$ and $\mathrm{H}-3^{\prime \prime \prime \prime}(\mathrm{J}=15.9 \mathrm{~Hz})$. Hence, 1 was established to be (3S)-O- $\alpha$-L-rhamnopyranosyl-(1 $\rightarrow 3)$-[4-O-(E )-coumaroyl ]- $\alpha$ L-rhamnopyranosyl-( $1 \rightarrow 6)-\beta$-D-glucopyranosyllinalool. This structure was supported by the FABMS data, which showed prominent fragments at m/z 639, 439, 293, and 147. Complete ${ }^{1} \mathrm{H}$ and ${ }^{13} \mathrm{C}$ NMR data (Table 1) assignment of 1, especially in the sugar region, was accomplished by analysis of two-dimensional COSY -45, NOESY, TOCSY, HMQC, and HMBC NMR spectra.

Compound $\mathbf{2}$ was assigned the same molecular formula as 1, as deduced from its FABMS and ${ }^{13} \mathrm{C}$ NMR data. It possessed spectroscopic data closely comparable to those of 1 except for those in the coumaroyl part of the molecule. Its ${ }^{1} \mathrm{H}$ NMR exhibited signals for two cis-conjugated olefinic protons at $\delta 5.77$ and $6.92(\mathrm{~J}=12.9 \mathrm{~Hz})$ in contrast to the trans-conjugated signals ( $\delta 6.33$ and 7.65, $\mathrm{J}=15.9 \mathrm{~Hz}$ ) observed for $\mathbf{1}$. Hence, 2 contained a cis-coumaroyl moiety, which was also supported by the evidence that $\mathrm{H}-5^{\prime \prime \prime \prime \prime}$ and H-9'"' appeared at a lower field in the ${ }^{1} \mathrm{H}$ NMR spectrum relative to those same signals in $\mathbf{1}$ (Table 1 ). ${ }^{8}$ Therefore, the structure of $\mathbf{2}$ was established as (3S)-O- $\alpha$-L-rhamnopyranosyl-( $1 \rightarrow 3)$-[4-O-(Z)-coumaroyl]- $\alpha$-L-rhamnopyranosyl-(1 $\rightarrow 6)-\beta$-D-glucopyranosyllinalool .

Compound $\mathbf{3}$ was assigned a molecular formula of $\mathrm{C}_{30} \mathrm{H}_{46} \mathrm{O}_{6}$, as deduced from the HREIMS and ${ }^{1} \mathrm{H}$ and ${ }^{13} \mathrm{C}$ NMR data, one more oxygen atom than that of 8. Its IR spectrum indicated the presence of a carbonyl $\left(1716 \mathrm{~cm}^{-1}\right)$, a carboxylic acid group (1688 $\left.\mathrm{cm}^{-1}\right)$, and a double bond $\left(1653 \mathrm{~cm}^{-1}\right)$. The ${ }^{1} \mathrm{H}$ N MR spectra of both $\mathbf{3}$ and $\mathbf{8}$ showed characteristic signals for the olefinic proton $\mathrm{H}-12(\delta 5.73$, brs), $\mathrm{H}-18$ ( $\delta 3.08, \mathrm{~s})$, and seven methyls including six singlets and one doublet $\left(\mathrm{H}_{3}-29\right)$, consistent with a $19 \alpha$ hydroxyursolic acid skeleton. ${ }^{20}$ In addition, the ${ }^{1} \mathrm{H}$ NMR spectrum of $\mathbf{3}$ exhibited two mutually coupled carbinoyl protons at $\delta 3.70$ (d) and 4.88 (d), instead of only one carbinoyl proton ( $\mathrm{H}-2)$ in $\mathbf{8}$, which appeared as a double doublet $(\delta 4.84, \mathrm{~J}=6.4,12.6 \mathrm{~Hz})$. This difference was also reflected in their ${ }^{13} \mathrm{C}$ NMR spectra, which indicated that a methylene signal $(C-1, \delta 50.6, \mathrm{t})$ in $\mathbf{8}$ was replaced by an oxygenated methine $(\delta 87.1, \mathrm{~d})$ in 3 . To account for this difference, $\mathrm{C}-1$ of $\mathbf{3}$ must be hydroxylated, and $\mathrm{OH}-1$ and $\mathrm{OH}-2$ should be $\beta$ - and $\alpha$-oriented, respectively, to fit the diaxial coupling constant between $\mathrm{H}-1$ and $\mathrm{H}-2 \mathrm{~J}=9.6$ $\mathrm{Hz}$ ). The NOESY spectrum of $\mathbf{3}$ exhibited mutual correlations between $\mathrm{H}-2, \mathrm{H}_{3}-24$, and $\mathrm{H}_{3}-25$, confirming $\mathrm{H}-2$ to be axially oriented. The HMBC spectrum revealed a threebond coupling of $\mathrm{H}_{3}-25(\delta 1.43)$ to $\mathrm{C}-1$ ( $\delta$ 87.1), which was directly coupled to $\mathrm{H}-1$, as observed in the HMQC spectrum, confirming $\mathrm{C}-1$ to be hydroxylated. Furthermore, correlations between the carbonyl carbon $(\delta 214.1)$ and two methyl protons $\left(\delta 1.02, \mathrm{H}_{3}-24 ; \delta 1.24, \mathrm{H}_{3}-23\right.$ ) in the HMBC spectrum confirmed the ketone group to be located at the C-3 position. Further analysis of 2D NMR data allowed the complete assignments of the ${ }^{1} \mathrm{H}$ and ${ }^{13} \mathrm{C}$ NMR spectra of $\mathbf{3}$, and the results are listed in Table 2 . Thus, compound $\mathbf{3}$ was established as $1 \beta, 2 \alpha, 19 \alpha$-trihydroxy-3-oxo-12-ursen28-oic acid.

Compound 4 was assigned a molecular formula of $\mathrm{C}_{30} \mathrm{H}_{48} \mathrm{O}_{5}$, by HREIMS, the same as that of $\mathbf{9}$. Similar to that of 9, its ${ }^{1} \mathrm{H}$ NMR spectrum exhibited characteristic signals for a $19 \alpha$-hydroxyol eanol ic acid skel eton, ${ }^{20}$ namely, seven methyl singlets and signals for an olefinic $\mathrm{H}-12(\delta$ 5.57 , brs), and the mutually coupled $\mathrm{H}-18$ ( $\delta 3.64$, brs) and $\mathrm{H}-19$ ( $\delta 3.61, \mathrm{brs})$, as determined by the COSY-45 spectrum. Both spectra also displayed another two carbinoyl proton signals, however, having quite different coupling patterns. These two protons, $\mathrm{H}-2$ and $\mathrm{H}-3$, in 9 are diaxially oriented, thus having a large coupling constant $(J=9.5$ $\mathrm{Hz}$ ), with the corresponding signals in 4 exhibiting a much smaller coupling constant. In addition, the coupling con- 
Table 1. ${ }^{1} \mathrm{H}$ and ${ }^{13} \mathrm{C}$ NMR and $2 \mathrm{D}$ NMR Data for Compound $\mathbf{1}$ (methanol- $\mathrm{d}_{4}, 500 \mathrm{MHz}$ )

\begin{tabular}{|c|c|c|c|c|}
\hline position & $\delta_{\mathrm{C}}(\mathrm{ppm})$ mult. $^{\mathrm{a}}$ & $\delta_{\mathrm{H}}$ mult. $(\mathrm{J} / \mathrm{Hz})^{\mathrm{b}}$ & $\mathrm{HMBC}(\mathrm{H} \rightarrow \mathrm{C})$ & $\operatorname{NOESY}(\mathrm{H} \rightarrow \mathrm{H})$ \\
\hline \multicolumn{5}{|l|}{ linalool } \\
\hline 1 & $115.3 t$ & $5.21 \mathrm{~m}$ & 2,3 & 2 \\
\hline 2 & $144.4 \mathrm{~d}$ & $6.06 \mathrm{dd}(10.7,18.0)$ & $3,4,9$ & 1 \\
\hline 3 & $81.4 \mathrm{~s}$ & & & \\
\hline 4 & $41.8 \mathrm{t}$ & $1.62 \mathrm{~m}$ & $2,3,6,9$ & \\
\hline 5 & $23.7 t$ & $2.04 \mathrm{~m}$ & $3,4,6,7$ & \\
\hline 6 & $125.7 \mathrm{~d}$ & $5.08 \mathrm{t}(7.0)$ & $4,5,8,10$ & \\
\hline 7 & $132.1 \mathrm{~s}$ & & & \\
\hline 8 & $17.8 \mathrm{q}$ & $1.56 \mathrm{~s}$ & $6,7,10$ & \\
\hline 9 & $23.6 q$ & $1.34 \mathrm{~s}$ & $2,3,4$ & \\
\hline 10 & $25.9 q$ & $1.61 \mathrm{~s}$ & $6,7,8$ & \\
\hline \multicolumn{5}{|l|}{ 3-O-glc } \\
\hline $1^{\prime}$ & $99.2 \mathrm{~d}$ & $4.33 \mathrm{~d}(7.8)$ & $2^{\prime}, 3^{\prime}, 3$ & $2^{\prime}, 3^{\prime}, 5^{\prime}$ \\
\hline $2^{\prime}$ & $75.1 \mathrm{~d}$ & $3.18 \mathrm{dd}(7.8,8.9)$ & $1^{\prime}, 3^{\prime}$ & $1^{\prime}$ \\
\hline $3^{\prime}$ & $78.3 \mathrm{~d}$ & 3.32 & $2^{\prime}, 4^{\prime}$ & $1^{\prime}$ \\
\hline $4^{\prime}$ & $71.7 \mathrm{~d}$ & 3.27 & $3^{\prime}, 5^{\prime}$ & \\
\hline $5^{\prime}$ & $76.4 \mathrm{~d}$ & 3.27 & $3^{\prime}, 4^{\prime}, 6^{\prime}$ & $l^{\prime}, 6^{\prime} a, 6^{\prime} b$ \\
\hline $6^{\prime} a$ & $68.0 \mathrm{t}$ & $3.56 \mathrm{dd}(6.0,10.4)$ & $1^{\prime \prime}, 5^{\prime}$ & $1^{\prime \prime}, 5^{\prime}, 6^{\prime} \mathrm{b}$ \\
\hline$\sigma^{\prime} b$ & & $3.93 \mathrm{dd}(3.6,10.4)$ & $1^{\prime \prime}, 5^{\prime}$ & $1^{\prime \prime}, 5^{\prime}, 6^{\prime} a$, \\
\hline \multicolumn{5}{|l|}{ 6'-O-rha } \\
\hline $1^{\prime \prime}$ & $101.9 \mathrm{~d}$ & 4.77 brs & $2^{\prime \prime}, 3^{\prime \prime}, 5^{\prime \prime}, 6^{\prime}$ & $2^{\prime \prime}, 5^{\prime \prime}, 6^{\prime} a, 6^{\prime} b$ \\
\hline $2^{\prime \prime}$ & $72.0 \mathrm{~d}$ & 3.98 & $3^{\prime \prime}, 4^{\prime \prime}$ & $1^{\prime \prime}$ \\
\hline $3^{\prime \prime}$ & $78.5 \mathrm{~d}$ & 4.00 & $1^{\prime \prime \prime} 4^{\prime \prime}$ & $1^{\prime \prime \prime}, 4^{\prime \prime}, 5^{\prime \prime \prime}$ \\
\hline $4^{\prime \prime}$ & $74.2 \mathrm{~d}$ & $5.18 \mathrm{t}(9.6)$ & $1^{\prime \prime \prime \prime}, 3^{\prime \prime}, 5^{\prime \prime}, 6^{\prime \prime}$ & $3^{\prime \prime}, 5^{\prime \prime}, 6^{\prime \prime}$ \\
\hline $5^{\prime \prime}$ & $67.9 \mathrm{~d}$ & $3.95 \mathrm{dq}(9.6,6.2)$ & $4^{\prime \prime}, 3^{\prime \prime}, 6^{\prime \prime}$ & $1^{\prime \prime}, 4^{\prime \prime}, 6^{\prime \prime}$ \\
\hline $6^{\prime \prime}$ & $17.9 \mathrm{q}$ & $1.16 \mathrm{~d}(6.2)$ & $4^{\prime \prime}, 5^{\prime \prime}$ & $4^{\prime \prime}, 5^{\prime \prime}$ \\
\hline \multicolumn{5}{|l|}{ 3"-O-rha } \\
\hline $1^{\prime \prime \prime}$ & $104.1 \mathrm{~d}$ & $4.82 \mathrm{~d}(1.5)$ & $3^{\prime \prime}, 3^{\prime \prime \prime}, 5^{\prime \prime \prime}$ & $2^{\prime \prime \prime}, 3^{\prime \prime}$ \\
\hline $2^{\prime \prime \prime}$ & $72.4 \mathrm{~d}$ & $3.68 \mathrm{dd}(1.5,3.3)$ & $3^{\prime \prime \prime}, 4^{\prime \prime \prime}$ & $1^{\prime \prime \prime}$ \\
\hline $3^{\prime \prime \prime}$ & $72.1 \mathrm{~d}$ & $3.70 \mathrm{dd}(3.3,9.4)$ & $4^{\prime \prime \prime}$ & $4^{\prime \prime \prime}$ \\
\hline $4^{\prime \prime \prime}$ & $73.9 \mathrm{~d}$ & 3.35 & $2^{\prime \prime \prime}, 6^{\prime \prime \prime}$ & $3^{\prime \prime \prime}, 5^{\prime \prime \prime}, 6^{\prime \prime \prime}$ \\
\hline $5^{\prime \prime \prime}$ & $70.2 d$ & $3.77 \mathrm{dq}(9.4,6.2)$ & $3^{\prime \prime \prime}, 4^{\prime \prime \prime}, 6^{\prime \prime \prime}$ & $3 " 4^{\prime \prime \prime}$ \\
\hline $6^{\prime \prime \prime}$ & $18.1 \mathrm{q}$ & $1.24 \mathrm{~d}(6.2)$ & $4^{\prime \prime \prime}, 5^{\prime \prime \prime}$ & $4^{\prime \prime \prime}, 5^{\prime \prime \prime}$ \\
\hline \multicolumn{5}{|l|}{ 4"-coumaroyl } \\
\hline $1^{\prime \prime \prime \prime}$ & $168.4 \mathrm{~s}$ & & & \\
\hline $2^{\prime \prime \prime \prime}$ & $114.6 \mathrm{~d}$ & $6.33 d(15.9)$ & $1^{\prime \prime \prime \prime \prime}, 3^{\prime \prime \prime \prime}, 4^{\prime \prime \prime \prime \prime}$ & $3^{\prime \prime \prime \prime}, 5^{\prime \prime \prime \prime}, 9^{\prime \prime \prime \prime}$ \\
\hline $3^{\prime \prime \prime \prime}$ & $147.4 d$ & $7.65 d(15.9)$ & $1^{\prime \prime \prime \prime}, 2^{\prime \prime \prime \prime}, 4^{\prime \prime \prime \prime}, 5^{\prime \prime \prime \prime}, 9^{\prime \prime \prime \prime}$ & $2^{\prime \prime \prime \prime}, 5^{\prime \prime \prime \prime \prime}, 9^{\prime \prime \prime \prime}$ \\
\hline $4^{\prime \prime \prime \prime}$ & $127.1 \mathrm{~s}$ & & & \\
\hline $5^{\prime \prime \prime \prime \prime}$ & $131.4 \mathrm{~d}$ & $7.47 d(8.6)$ & $3^{\prime \prime \prime \prime}, 6^{\prime \prime \prime \prime}, 7^{\prime \prime \prime \prime}, 8^{\prime \prime \prime \prime}, 9^{\prime \prime \prime \prime}$ & $2^{\prime \prime \prime \prime}, 3^{\prime \prime \prime \prime}, 6^{\prime \prime \prime \prime}$ \\
\hline $6^{\prime \prime \prime \prime}$ & $116.8 \mathrm{~d}$ & $6.80 \mathrm{~d}(8.6)$ & $4^{\prime \prime \prime \prime}, 7^{\prime \prime \prime \prime \prime}, 8^{\prime \prime \prime \prime \prime}$ & $5^{\prime \prime \prime \prime \prime}$ \\
\hline $7^{\prime \prime \prime \prime}$ & $161.5 \mathrm{~s}$ & & & \\
\hline $8^{\prime \prime \prime \prime}$ & $116.8 \mathrm{~d}$ & $6.80 \mathrm{~d}(8.6)$ & $4^{\prime \prime \prime \prime}, 6^{\prime \prime \prime \prime}, 7^{\prime \prime \prime \prime}$ & $9^{\prime \prime \prime \prime \prime}$ \\
\hline $9^{\prime \prime \prime \prime}$ & $131.4 \mathrm{~d}$ & $7.47 \mathrm{~d}(8.6)$ & , ',"', & $2^{\prime \prime \prime \prime}, 3^{\prime \prime \prime \prime \prime}, 8^{\prime \prime \prime \prime}$ \\
\hline
\end{tabular}

a Multiplicities were obtained from DEPT experiments. ${ }^{b}$ Signals without multiplicity were picked up from COSY -45 or HMQC spectra.

stants between $\mathrm{H}-2$ and $\mathrm{H}_{2}-1$ were found to be 12.1 and $3.9 \mathrm{~Hz}$, corresponding to a diaxial and an axial-equatorial coupling, respectively. Thus, $\mathrm{H}-2$ was axially oriented. Consequently, $\mathrm{H}-3$ was deduced to be equatorially oriented. In the NOESY spectrum, the signal of $\mathrm{H}-2$ showed correlations with those of $\mathrm{H}-3, \mathrm{H}_{3}-24$, and $\mathrm{H}_{3}-25$, confirming both $\mathrm{H}-2$ and $\mathrm{H}-3$ to be $\beta$-oriented. I ts $\mathrm{HMBC}$ spectrum displayed the correlation of $\mathrm{H}-3$ to $\mathrm{C}-1, \mathrm{C}-2, \mathrm{C}-4, \mathrm{C}-5, \mathrm{C}-23$, and $\mathrm{C}-24$, confirming $\mathrm{H}-3$ to be hydroxylated. Further analysis of these 2D NMR data allowed the complete assignment of its ${ }^{1} \mathrm{H}$ and ${ }^{13} \mathrm{C}$ NMR spectra, and the results are listed in Table 2. Accordingly, compound $\mathbf{4}$ was assigned as $2 \alpha, 3 \alpha, 19 \alpha$-trihydroxy-12-oleanen-28-oic acid.

The inflammatory response is a nonspecific immune response triggered by pathogenic microorganism infection or tissue injury and provides early protection in restricting the tissue damage to the site of infection or tissue injury. ${ }^{21}$ Several immune cells including lymphocytes, neutrophils, monocytes, eosinophils, and basophils are involved in the inflammatory response, which in response to invasive organisms, if sufficiently intense or inappropriately prolonged, could paradoxically aggravate the injury or even cause death. The use of antiinflammatory medications must therefore be discreet. Blockade of the Iymphocyte activation and proliferation is an antiinflammatory mechanism. ${ }^{22}$ In this investigation the isolated pure compounds from E. deflexa were tested for their antiproliferation activity on human mononuclear cells involving $T$ Iymphocytes, B lymphocytes, and macrophages isolated from peripheral blood. ${ }^{23}$ Compounds $3,5,6,7$, and $\mathbf{8}$, having $I C_{50}$ values of $26.9,28.8,32.5,38.1$, and $40.0 \mu \mathrm{M}$, respectively, were found to be moderately active in contrast to cydosporin $\mathrm{A}$, with a $\mathrm{IC}_{50}$ value of $0.012 \mu \mathrm{M}$. However, compounds $\mathbf{1}$ and $\mathbf{2}$ did not show any activity up to 100 $\mu \mathrm{M}$ concentration. The quantities of $\mathbf{4}$ and $\mathbf{9}$ obtained were insufficient to perform this biological investigation.

\section{Experimental Section}

General Experimental Procedures. Optical rotations were measured using a J ASCO DIP-180 digital spectropolarimeter. UV spectra were measured in $\mathrm{MeOH}$ on a Hitachi U-2000 spectrophotometer. The IR spectra were recorded on a Nicolet 510P FT-IR spectrometer. The NMR spectra were recorded in $\mathrm{MeOH}-\mathrm{d}_{4}$ or pyridine- $\mathrm{d}_{5}$ at room temperature on a Bruker DMX-500 SB spectrometer, and the solvent resonances were used as internal shift references. The 2D NMR spectra were recorded using standard pulse sequences. FABMS were recorded on a JEOL SX-102A instrument using $\mathrm{m}$ nitrobenzyl alcohol (NBA) as the matrix. Sephadex LH-20 (Pharmacia Biotech) was used for open col umn chromatography. TLC was performed using silica gel $60 \mathrm{~F}_{254}$ plates (200 $\mu \mathrm{m}$, Merck). HPLC was performed using an ODS column (Hyperprep ODS, 10 mm i.d. × 250 mm, Keystone Scientific 
Table 2. ${ }^{1 \mathrm{H}}$ and ${ }^{13} \mathrm{C}$ NMR Data for Compounds $\mathbf{3}$ and $\mathbf{4}$ (pyridine- $\mathrm{d}_{5}, 500 \mathrm{MHz}$ )

\begin{tabular}{|c|c|c|c|c|}
\hline \multirow[b]{2}{*}{ position } & \multicolumn{2}{|c|}{3} & \multicolumn{2}{|c|}{4} \\
\hline & $\delta_{C}(p p m)$ mult. $^{a}$ & $\delta_{\mathrm{H}}$ mult. $(\mathrm{J} / \mathrm{Hz})^{\mathrm{b}}$ & $\delta_{\mathrm{C}}(\mathrm{ppm})$ mult. $^{\mathrm{a}}$ & $\delta_{\mathrm{H}}$ mult. $(\mathrm{J} / \mathrm{Hz})^{\mathrm{b}}$ \\
\hline 1 & $87.1 \mathrm{~d}$ & $3.70 \mathrm{~d}(9.6)$ & $43.1 \mathrm{t}$ & $\begin{array}{l}1.80 \mathrm{t}(12.1) \\
1.95 \mathrm{dd}(3.9,12.1)\end{array}$ \\
\hline 2 & $76.4 \mathrm{~d}$ & $4.88 d(9.6)$ & $66.6 \mathrm{~d}$ & $4.34 \mathrm{dt}(3.9,12.1)$ \\
\hline 3 & $214.1 \mathrm{~s}$ & & $79.9 \mathrm{~d}$ & $3.79 \mathrm{brs}$ \\
\hline 4 & $48.5 \mathrm{~s}$ & & $39.4 \mathrm{~s}$ & \\
\hline 5 & $53.9 \mathrm{~d}$ & 1.16 & $49.4 d$ & 1.69 \\
\hline 6 & $19.5 \mathrm{t}$ & $\begin{array}{l}1.52 \mathrm{~m} \\
1.59 \mathrm{~m}\end{array}$ & $19.1 \mathrm{t}$ & $\begin{array}{l}1.42 \mathrm{~m} \\
1.58 \mathrm{~m}\end{array}$ \\
\hline 7 & $33.9 \mathrm{t}$ & $\begin{array}{l}1.41 \mathrm{~m} \\
1.57 \mathrm{~m}\end{array}$ & $33.8 \mathrm{t}$ & $\begin{array}{l}1.39 \mathrm{~m} \\
1.61 \mathrm{~m}\end{array}$ \\
\hline 8 & $41.5 \mathrm{~s}$ & & $40.7 \mathrm{~s}$ & \\
\hline 9 & $48.9 \mathrm{~d}$ & $2.23 \mathrm{dd}(7.4,10.8)$ & $48.7 \mathrm{~d}$ & 2.13 \\
\hline 10 & $44.0 \mathrm{~s}$ & & $39.3 \mathrm{~s}$ & \\
\hline 11 & $28.4 \mathrm{t}$ & $\begin{array}{l}2.65 \mathrm{~m} \\
3.28 \mathrm{~m}\end{array}$ & $24.7 \mathrm{t}$ & $2.12 \mathrm{~m}$ \\
\hline 12 & $129.8 d$ & $5.73 \mathrm{brs}$ & $124.0 \mathrm{~d}$ & $5.57 \mathrm{brs}$ \\
\hline 13 & $139.4 \mathrm{~s}$ & & $145.4 \mathrm{~s}$ & \\
\hline 14 & $42.6 \mathrm{~s}$ & & $42.7 \mathrm{~s}$ & \\
\hline 15 & $29.9 t$ & $\begin{array}{l}1.31 \mathrm{~m} \\
2.35 \mathrm{~m}\end{array}$ & $29.6 \mathrm{t}$ & $2.10-2.20$ \\
\hline 16 & $26.9 \mathrm{t}$ & $\begin{array}{l}2.10 \mathrm{~m} \\
3.15 \mathrm{~m}\end{array}$ & $28.9 t$ & $\begin{array}{l}2.16 \mathrm{~m} \\
2.83 \mathrm{~m}\end{array}$ \\
\hline 17 & $48.8 \mathrm{~s}$ & & $46.5 \mathrm{~s}$ & \\
\hline 18 & $55.0 \mathrm{~d}$ & $3.08 \mathrm{~s}$ & $45.3 \mathrm{~d}$ & 3.64 brs \\
\hline 19 & $73.1 \mathrm{~s}$ & & $81.7 \mathrm{~d}$ & $3.61 \mathrm{brs}$ \\
\hline 20 & $42.8 d$ & $1.50 \mathrm{~m}$ & $36.2 \mathrm{~s}$ & \\
\hline 21 & $27.4 \mathrm{t}$ & $\begin{array}{l}1.36 \mathrm{~m} \\
2.12 \mathrm{~m}\end{array}$ & $29.7 t$ & $2.10-2.20$ \\
\hline 22 & $38.9 t$ & $\begin{array}{l}2.08 \mathrm{~m} \\
2.18 \mathrm{~m}\end{array}$ & $34.2 \mathrm{t}$ & $\begin{array}{l}2.05 \mathrm{~m} \\
2.21 \mathrm{~m}\end{array}$ \\
\hline 23 & $25.9 q$ & $1.24 \mathrm{~s}$ & $29.9 q$ & $1.28 \mathrm{~s}$ \\
\hline 24 & $22.1 \mathrm{q}$ & $1.02 \mathrm{~s}$ & $22.7 q$ & $0.93 \mathrm{~s}$ \\
\hline 25 & $13.3 q$ & $1.43 \mathrm{~s}$ & $17.0 \mathrm{q}$ & $1.02 \mathrm{~s}$ \\
\hline 26 & $18.2 \mathrm{q}$ & $1.23 \mathrm{~s}$ & $18.1 \mathrm{q}$ & $1.09 \mathrm{~s}$ \\
\hline 27 & $25.2 \mathrm{q}$ & $1.70 \mathrm{~s}$ & $25.3 q$ & $1.39 \mathrm{~s}$ \\
\hline 28 & $181.2 \mathrm{~s}$ & & $181.5 \mathrm{~s}$ & \\
\hline 29 & $17.2 \mathrm{q}$ & $1.13 \mathrm{~d}(6.5)$ & $29.3 q$ & $1.20 \mathrm{~s}$ \\
\hline 30 & $27.5 q$ & $1.45 \mathrm{~s}$ & $25.3 \mathrm{q}$ & $1.13 \mathrm{~s}$ \\
\hline
\end{tabular}

a Multiplicities were obtained from DEPT experiments. ${ }^{b}$ Signals without multiplicity were picked up from COSY-45 or HMQC spectra.

Inc., Bellefonte, PA; detector, UV $254 \mathrm{~nm}$ ) and a silica column (Hyperprep HS silica, $10 \mathrm{~mm}$ i.d. $\times 250 \mathrm{~mm}$, ThermoQuest Hypersil, Runcorn, UK; detector, RI). GLC: HP gas chromatograph 6890; MS, HP 5973 mass selective detector; column, HP-5MS fused silica capillary column, $0.25 \mathrm{~mm}$ i.d. $\times 30 \mathrm{~m}$, carrier gas, $\mathrm{He}$; flow rate, $1 \mathrm{~mL} / \mathrm{min}$.

Plant Material. Leaves of E. deflexa were collected from Central Cross Island Road in central Taiwan in April 2000 and were identified by Chii-Cheng Liao, a Ph.D. candidate in the Department of Botany of National Taiwan University. Voucher specimens (No. 20000405) have been deposited at the Institute of Botany, Academia Sinica, Nankang, Taipei, Taiwan.

Extraction and Isolation. Dried leaves (500 g) were ground into a powder and extracted three times with $500 \mathrm{~mL}$ of $\mathrm{MeOH}$ for 2 days. The methanolic extract was adjusted to $85 \%$ in aqueous solution for an n-hexane partition, which generated two fractions soluble in methanol and n-hexane. Subsequently, the methanol-soluble fraction was then vacuumevaporated to dryness ( $75 \mathrm{~g}$ ) and further partitioned between chloroform and water, and the remaining water solution was extracted three times with n-butanol. Both the n-butanol and chl or oform layers were evaporated to dryness and redisssolved in $\mathrm{MeOH}$ for individual chromatographic separation. The first separation step was carried out using gel filtration chromatography on a Sephadex L H-20 column $(3 \times 55 \mathrm{~cm})$ and eluted by $\mathrm{MeOH}$ with a flow rate of $13 \mathrm{~mL} / \mathrm{min}$.

Fractions collected from the n-butanol layer were checked for their compositions by TLC using $\mathrm{CH}_{2} \mathrm{Cl}_{2}-\mathrm{EtOAC}-\mathrm{HCO}_{2} \mathrm{H}-$ $\mathrm{H}_{2} \mathrm{O}$ (15:70:5:1) for development. Vanillin-sulfuric acid (yellow-green spots) and observation under long-wave UV were used in the detection of monoterpene glycosides. Subsequently, the monoterpene glycoside fractions from the above separation were combined and rechromatographed on a reversed-phase HPLC column with $\mathrm{MeCN}-\mathrm{H}_{2} \mathrm{O}(40: 60)$ as eluent to yield $\mathbf{1}$ (360 mg) and 2 (195 mg).

Fractions collected from the chloroform layer were further purified by repetitive HPLC separations on a Hyperprep ODS column with $\mathrm{MeCN}-\mathrm{H}_{2} \mathrm{O}(60: 40)$ as eluent to give subfractions 1 and 2. Subsequently, HPLC of subfraction 1 on a silica col umn with $\mathrm{CH}_{2} \mathrm{Cl}_{2}$-EtOAc (3:7) as eluent afforded $\mathbf{3}$ (6 mg), 4 (4 mg), 5 (10.8 mg), 6 (12 mg), and 9 (3.5 mg). Subfraction 2 was purified by using the same col umn with n-hexane-EtOAc (2:1) as eluent to yield 7 (12 $\mathrm{mg})$ and 8 (14 mg).

(3S)-0- $\alpha-L-r h a m n o p y r a n o s y l-(1 \rightarrow 3)-[4-0-(E)-c o u m a r o y l]-$ $\alpha-L-r h a m n o p y r a n o s y l-(1 \rightarrow 6)-\beta$-D-glucopyranosyllinaIool (1): amorphous white powder; $\mathrm{mp} 144-146{ }^{\circ} \mathrm{C}$; $[\alpha]^{25} \mathrm{D}$ $-31.8^{\circ}$ (c 0.67, MeOH); IR (KBr) $v_{\max } 3413,1605,1169,1046$ $\mathrm{cm}^{-1}$; UV $\lambda_{\max }(\mathrm{MeOH})(\log \epsilon) 203$ (4.0), 227 (3.9), 313 (4.2) $\mathrm{nm} ;{ }^{1} \mathrm{H}$ and ${ }^{13} \mathrm{C}$ NMR data, see Table $1 ;$ FABMS m/z [M + $\mathrm{Na}^{+} 777$ (20), 639 (4), 585 (3), 439 (17), 293 (47), 154 (48), 147 (100), 136 (37), 82 (35), 69 (56); HRFABMS m/z [M + H] $]^{+}$ 755.3472 (calcd for $\mathrm{C}_{37} \mathrm{H}_{55} \mathrm{O}_{16}$ 755.3490); $\mathrm{R}_{\mathrm{f}} 0.32$ [EtOAc$\left.\mathrm{HCO}_{2} \mathrm{H}-\mathrm{H}_{2} \mathrm{O}(85: 10: 15)\right]$.

(3S)-O- $\alpha$-L-rhamnopyranosyl-(1 $\rightarrow 3)-[4-0-(Z)-c o u m a r o y l]-$ $\alpha$-L-rhamnopyranosyl-( $\rightarrow 6)-\beta$-D-glucopyranosyllinaIool (2): amorphous white powder; mp $136-137{ }^{\circ} \mathrm{C}$; $[\alpha]^{25} \mathrm{D}$ $-87.4^{\circ}$ (c $\left.0.39, \mathrm{MeOH}\right)$; IR ( $\mathrm{KBr}$ ) $v_{\max } 3401,1605,1163,1046$ $\mathrm{cm}^{-1}$; UV $\lambda_{\max }(\mathrm{MeOH})(\log \epsilon) 203(4.1), 312(4.1) \mathrm{nm} ;{ }^{1} \mathrm{H}$ NMR $\left(\mathrm{CD}_{3} \mathrm{OD}\right) \delta 7.69\left(2 \mathrm{H}, \mathrm{d}, \mathrm{J}=8.7 \mathrm{~Hz}, \mathrm{H}-5^{\prime \prime \prime \prime},-9^{\prime \prime \prime \prime}\right), 6.92(1 \mathrm{H}, \mathrm{d}$, $\left.\mathrm{J}=12.9 \mathrm{~Hz}, \mathrm{H}-3^{\prime \prime \prime \prime}\right), 6.76\left(2 \mathrm{H}, \mathrm{d}, \mathrm{J}=8.7 \mathrm{~Hz}, \mathrm{H}-6^{\prime \prime \prime \prime},-8^{\prime \prime \prime \prime}\right)$, $6.04(1 \mathrm{H}, \mathrm{dd}, \mathrm{J}=10.7,18.0 \mathrm{~Hz}, \mathrm{H}-2), 5.77(1 \mathrm{H}, \mathrm{d}, \mathrm{J}=12.9$ 
$\left.\mathrm{Hz}, \mathrm{H}-2^{\prime \prime \prime \prime}\right), 5.20\left(2 \mathrm{H}, \mathrm{m}, \mathrm{H}_{2}-1\right), 5.12\left(1 \mathrm{H}, \mathrm{t}, \mathrm{J}=9.8 \mathrm{~Hz}, \mathrm{H}-4^{\prime \prime}\right)$, $5.09(1 \mathrm{H}, \mathrm{t}, \mathrm{J}=7.3 \mathrm{~Hz}, \mathrm{H}-6), 4.79\left(1 \mathrm{H}, \mathrm{brs}, \mathrm{H}-\mathrm{1}^{\prime \prime \prime}\right), 4.75(1 \mathrm{H}$, brs, $\left.\mathrm{H}-1^{\prime \prime}\right), 4.32\left(1 \mathrm{H}, \mathrm{d}, \mathrm{J}=7.8 \mathrm{~Hz}, \mathrm{H}-\mathrm{I}^{\prime}\right), 3.95\left(1 \mathrm{H}, \mathrm{H}-2^{\prime \prime}\right), 3.92$ $\left(1 \mathrm{H}, \mathrm{dd}, \mathrm{J}=3.3,10.5 \mathrm{~Hz}, \mathrm{H}-6^{\prime} \mathrm{b}\right), 3.90\left(1 \mathrm{H}, \mathrm{H}-3^{\prime \prime}\right), 3.86(1 \mathrm{H}$, $\left.\mathrm{dq}, \mathrm{J}=9.8,6.2 \mathrm{~Hz}, \mathrm{H}-5^{\prime \prime}\right), 3.77\left(1 \mathrm{H}, \mathrm{dq}, \mathrm{J}=9.4,6.3 \mathrm{~Hz}, \mathrm{H}-5^{\prime \prime \prime}\right)$, $3.71\left(1 \mathrm{H}, \mathrm{H}-2^{\prime \prime \prime}\right), 3.70\left(1 \mathrm{H}, \mathrm{H}-3^{\prime \prime \prime}\right), 3.54(1 \mathrm{H}, \mathrm{dd}, \mathrm{J}=6.2,10.5$ $\left.\mathrm{Hz}, \mathrm{H}-6^{\prime} \mathrm{a}\right), 3.32\left(1 \mathrm{H}, \mathrm{H}-4^{\prime \prime \prime}\right), 3.31\left(1 \mathrm{H}, \mathrm{H}-2^{\prime}\right), 3.26\left(1 \mathrm{H}, \mathrm{H}-5^{\prime}\right)$, $3.23\left(1 \mathrm{H}, \mathrm{H}-4^{\prime}\right), 3.17\left(1 \mathrm{H}, \mathrm{dd}, \mathrm{J}=7.9,9.0 \mathrm{~Hz}, \mathrm{H}-2^{\prime}\right), 2.04(2 \mathrm{H}$ $\left.\mathrm{m}, \mathrm{H}_{2}-5\right), 1.65\left(3 \mathrm{H}, \mathrm{s}, \mathrm{H}_{3}-10\right), 1.61\left(2 \mathrm{H}, \mathrm{m}, \mathrm{H}_{2}-4\right), 1.58(3 \mathrm{H}, \mathrm{s}$, $\left.\mathrm{H}_{3}-8\right), 1.33\left(3 \mathrm{H}, \mathrm{s}, \mathrm{H}_{3}-9\right), 1.24\left(3 \mathrm{H}, \mathrm{d}, \mathrm{J}=6.2 \mathrm{~Hz}, \mathrm{H}_{3}-6^{\prime \prime \prime}\right), 1.16$ $\left(3 \mathrm{H}, \mathrm{d}, \mathrm{J}=6.2 \mathrm{~Hz}, \mathrm{H}_{3}-6^{\prime \prime}\right) ;{ }^{13} \mathrm{C} N M R\left(\mathrm{CD}_{3} \mathrm{OD}\right) \delta 167.2\left(\mathrm{~s}, \mathrm{C}-1^{\prime \prime \prime \prime}\right)$,

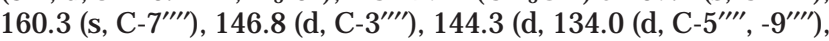
132.1 (s, C-7), 127.5 (s, C-4"'"'), 125.8 (d, C-6, 115.9 (d, C-6"'”, $\left.-8^{\prime \prime \prime \prime}\right), 115.8$ (d, C-2"'”), 115.3 (t, C-1), 104.1 (d, C-1"'), 101.9 (d, C- $\left.1^{\prime \prime}\right), 99.2\left(d, C-1^{\prime}\right), 81.4(s, C-3), 78.6\left(d, C-3^{\prime \prime}\right), 78.3\left(d, C-3^{\prime}\right)$, 76.5 (d, C-5'), 75.1 (d, C-2'), 73.9 (d, C-4"'), 73.8 (d, C-4'), 72.5 (d, C-2'"), 72.1 (d, C-3"'), 72.0 (d, C-2'), 71.9 (d, C-4'), 70.2 (d, C-5'”) 68.2 (t, C-6'), 68.0 (d, C-5"), 41.8 (t, C-4), 25.9 (q, C-10), 23.7 (t, C-5), 23.6 (q, C-9), 18.1 (q, C-6"'), 18.0 (q, C-6") 17.8 (q, C-8); FABMS m/z [M + Na] ${ }^{+} 777(23), 639$ (3), 439 (10), 293 (25), 176 (14), 154 (52), 147 (80), 136 (40), 82 (48), 69 (100); HRFABMS $\mathrm{m} / \mathrm{z}[\mathrm{M}+\mathrm{H}]^{+} 755.3478$ (calcd for $\mathrm{C}_{37} \mathrm{H}_{55} \mathrm{O}_{16}$ 755.3490); $\mathrm{R}_{\mathrm{f}} 0.38$ [EtOAc- $\mathrm{HCO}_{2} \mathrm{H}-\mathrm{H}_{2} \mathrm{O}$ (85:10:15)].

$1 \beta, 2 \alpha, 19 \alpha-T r i h y d r o x y-3-0 x 0-12-u r s e n-28-o i c$ acid (3): amorphous white powder; $\mathrm{mp} 218-220{ }^{\circ} \mathrm{C}$; $[\alpha]^{25} \mathrm{D}+29.7^{\circ}$ (c $0.59, \mathrm{MeOH})$; I R (KBr) $v_{\max } 2934,1716,1688,1653,1558,1541$, $1456 \mathrm{~cm}^{-1} ;{ }^{1} \mathrm{H}$ and ${ }^{13} \mathrm{C}$ NMR data, see Table 2; FABMS m/z $[\mathrm{M}+\mathrm{Na}]^{+} 525$ (100), 439 (18), 307 (12), 233 (8), 187 (18), 154 (42), 119 (40), 91 (50), 56 (52); HREIMS m/z [M ] 502.3256 (calcd for $\mathrm{C}_{30} \mathrm{H}_{46} \mathrm{O}_{6}$ 502.3294); $\mathrm{R}_{\mathrm{f}} 0.5$ [EtOAC- $\mathrm{CH}_{2} \mathrm{Cl}_{2}$ (7:3)].

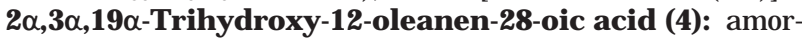
phous white powder; mp $215-217{ }^{\circ} \mathrm{C}$; $[\alpha]^{25} \mathrm{D}+19.6^{\circ}$ (c 0.1 , $\mathrm{MeOH}) ; \mathrm{IR}(\mathrm{KBr}) v_{\max } 2932,1693,1682,1454 \mathrm{~cm}^{-1} ;{ }^{1} \mathrm{H}$ and ${ }^{13} \mathrm{C}$ NMR data, see Table 2; FABMS m/z [M + Na] 511 (100), 407 (10), 246 (12), 201 (37), 187 (35), 145 (37), 119 (88), 91 (88), 56 (98); HREIMS m/z [M] 488.3470 (calcd for $\mathrm{C}_{30} \mathrm{H}_{48} \mathrm{O}_{5}$ 488.3502); $\mathrm{R}_{\mathrm{f}} 0.46$ [EtOAc- $\mathrm{CH}_{2} \mathrm{Cl}_{2}$ (7:3)].

Acid Hydrolysis of $\mathbf{1}$. Compound $\mathbf{1}(80 \mathrm{mg})$ was hydrolyzed by $2 \mathrm{~N} \mathrm{HCl}(10 \mathrm{~mL})$ at room temperature overnight. The reaction mixture was then extracted with $n$-hexane $(10 \mathrm{~mL} \times$ 3). The n-hexane layer was evaporated to give a residue, which was chromatographed by semipreparative HPLC (Hyperprep HS Silica, $250 \times 10 \mathrm{~mm}$ ) using n-hexane-EtOAc (2:1) as the eluent to give $\mathrm{S}-(+)$-linalool $(4.6 \mathrm{mg}):[\alpha]^{25} \mathrm{D}+2.7^{\circ}$ (c 0.23 , $\left.\mathrm{CHCl}_{3}\right)\left(\mathrm{lit}^{18}[\alpha]^{20}{ }_{\mathrm{D}}+19.2^{\circ}\right) ;{ }^{1} \mathrm{H} \mathrm{NMR}\left(500 \mathrm{MHz}, \mathrm{CDCl}_{3}\right) \delta 5.04$ $(1 \mathrm{H}, \mathrm{dd}, \mathrm{J}=1.2,10.8 \mathrm{~Hz}, \mathrm{H}-1 \mathrm{a}), 5.19(1 \mathrm{H}, \mathrm{dd}, \mathrm{J}=1.2,17.3$ $\mathrm{Hz}, \mathrm{H}-1 \mathrm{~b}), 5.89$ (1H, dd, J = 10.8, $17.3 \mathrm{~Hz}, \mathrm{H}-2), 1.55(2 \mathrm{H}, \mathrm{m}$, $\left.\mathrm{H}_{2}-4\right), 2.00\left(2 \mathrm{H}, \mathrm{m}, \mathrm{H}_{2}-5\right), 5.10(1 \mathrm{H}, \mathrm{t}, \mathrm{J}=6.5 \mathrm{~Hz}, \mathrm{H}-6), 1.58$ $\left(3 \mathrm{H}, \mathrm{S}, \mathrm{H}_{3}-8\right), 1.26\left(3 \mathrm{H}, \mathrm{S}, \mathrm{H}_{3}-9\right), 1.66\left(3 \mathrm{H}, \mathrm{s}, \mathrm{H}_{3}-10\right)$; GC-MS $\mathrm{m} / \mathrm{z} 154[\mathrm{M}]^{+} ; R_{\mathrm{f}} 0.47$ [n-hexane-EtOAc (2:1)].

Monosaccharide Composition Analysis of 1. Compound 1 (1 mg) was methanolyzed with $0.5 \mathrm{M}$ methanolic $\mathrm{HCl}$ at 80 ${ }^{\circ} \mathrm{C}$ for $16 \mathrm{~h}$. Evaporation of the reaction mixture gave a residue, which was treated with the Sylon HTP trimethylsilylation reagent (Supelco, Bell efonte, PA) for 20 min at room temperature. After removal of the excess reagent and organic solvent by condensation, the persilylated products were dissolved in n-hexane, and this solution was used for GC-MS analysis. GC-MS analysis of the trimethylsilylated derivatives was performed on a fused silica capillary column using a temperature gradient of 60 to $140{ }^{\circ} \mathrm{C}$ at $25^{\circ} \mathrm{C} / \mathrm{min}$, increased to 250 ${ }^{\circ} \mathrm{C}$ at $5{ }^{\circ} \mathrm{C} / \mathrm{min}$, and then increased to $300^{\circ} \mathrm{C}$ at $10^{\circ} \mathrm{C} / \mathrm{min}$. When compared with the authentic standards, the retention time of persilylated glucose and rhamnose was found to be 15.54 (15.89) and 10.08 (10.30) min, respectively.

Acknowledgment. This research was supported by grants to C.-H.C. from the National Science Council (NSC-89-2311B001-026) and a postdoctoral fellowship to T.-H.L. from Academia Sinica, Taiwan. We are grateful to Ms. Shoou-Ling Huang for the NMR data acquisition in the Instrumentation Center of the College of Science, National Taiwan University. We also want to thank Dr. Kay-Hooi Khoo (Institute of Biochemistry, Academia Sinica, Taipei, Taiwan) for the monosaccharide composition data.

\section{References and Notes}

(1) Liu, T. S. In Flora of Taiwan; Li, H. L., Liu, T. S., Huang, T. C. Koyama, T.;, DeVol, C. E., Eds.; Epoch Publishing Co., Ltd.: Taipei, Taiwan, 1977; Vol. 3; p 65.

(2) Chiu, N. Y., Chang, K. H., Eds. The Illustrated Medicinal Plants of Taiwan; SMC Publishing Inc.: Taipei, Taiwan, 1995; Vol. 4.

(3) Chiu, N. Y., Chang, K. H., Eds. Thelllustrated Medicinal Plants of Taiwan; SMC Publishing Inc.: Taipei, Taiwan, 1995; Vol. 5.

(4) de Tommasi, N.; Simone, F. D.; Aquino, R.; Pizza, C.; Liang, Z. Z. J . Nat. Prod. 1990, 53, 810-815.

(5) de Tommasi, N.; Aquino, R.; Simone, F. D.; Pizza, C. J . Nat. Prod 1992, 55, 1025-1032.

(6) Yanagisawa, H.; Ohshima, Y.; Okada, Y.; Takahashi, K.; Shibata, S Chem. Pharm. Bull. 1988, 36, 1270-1274.

(7) Shimizu, M.; Uemitsu, N.; Shirota, M.; Matsumoto, K.; Tezuka, Y. Chem. Pharm. Bull. 1996, 44, 2181-2182.

(8) de Tommasi, N.; Simone, F. D.; Pizza, C.; Mahmood, N.; Moore, P. S.; Conti, C.; Orsi, N.; Stein, M. L. J . Nat. Prod. 1992, 55, 10671073.

(9) Shimizu, M.; Fukumura, H.; Tsuji, H.; Tanaami, S.; Hayashi, T.; Morita, N. Chem. Pharm. Bull. 1986, 34, 2614-2617.

(10) de Tommasi, N.; Simone, F. D.; Cirino, G.; Cicala, C.; Pizza, C. Planta Med. 1991, 57, 414-416

(11) I varra, M. D.; Paya, N.; Villar, A. Planta Med. 1988, 54, 282-286.

(12) Fang, J.-M.; Wang, K.-C.; Cheng, Y.-S. Phytochemistry 1991, 30 3383-3387.

(13) Kashiwada, Y.; Wang, H.-K.; Nagao, T.; Kitanaka, S.; Yasuda, I.; Fujioka, T.; Yamagishi, T.; Cosentino, L. M.; Kozuka, M.; Okabe, H.; Ikeshiro, Y.; Hu, C.-Q.; Yeh, E.; Lee, K.-H. J. Nat. Prod. 1998, 61 1090-1095.

(14) Xu, H.-X.; Zeng, F.-Q.; Wan, M.; Sim, K.-Y. J . Nat. Prod. 1996, 59 $643-645$.

(15) Anjaneyulu, A. S. R.; Rama Prasad, A. V. Phytochemistry 1982, 21 2057-2060

(16) Conrad, J .; Vogler, B.; Klaiber, I.; Roos, G.; Walter, U.; Kraus, W. Phytochemistry 1998, 48, 647-650.

(17) Crews, P.; Rodríguez, J .; J aspars, M. Organic Structure Analysis Oxford University Press: New York, 1998.

(18) Weast, R. C., Grasselli, J. G., Eds. Handbook of Data on Organic Compounds; CRC Press: Boca Raton, FL, 1989; Vol. 5.

(19) Sweeley, C. C.; Bentley, R.; Makita, M.; Wells, W. W. J . Am. Chem Soc. 1963, 85, 2497-2507.

(20) Doddrell, D. M.; Khong, P. W.; Lewis, K. G. Tetrahedron Lett. 1974 $27,2381-2384$

(21) J aneway, C. A.; Travers, P.; Hunt, S.; Walport, M. Immunobiology: The Immune System in Health and Disease; Garland Publishing Inc: New York, 1997.

(22) Adams, D. O.; Hamilton, T. A. Annu. Rev. Immunol. 1984, 2, 283318.

(23) Lin, L. C.; Kuo, Y. C.; Chou, C. J . J . Nat. Prod. 1999, 62, 405-408. NP0100237 